\title{
Major venous thrombosis: a complication of transvenous pacemaker electrodes
}

\author{
HENRYK KRUG, FRANCISZEK ZERBE \\ From the Department of Cardiology, Medical Academy, Poznan, Poland
}

SUMMARY Two patients with transvenous permanent pacemakers developed thrombosis and occlusion of the superior vena cava around a broken pacing wire, with clot extending into the innominate, subclavian, and axillary veins. Both patients had symptoms and signs of the superior vena cava syndrome which failed to resolve despite anticoagulant and thrombolytic treatment. Obstruction to venous flow, however, was relieved in large measure by the development of collateral venous channels. One of the patients has shown mild symptoms for two years and the other for two months of follow-up. It is suggested that anticoagulant prophylaxis should be given to all patients with a broken wire which cannot easily be removed.

Common complications of permanent transvenous pacing include displacement of electrodes from the original contact points, infection at the site of the generator and of the wire track, cardiac perforation, an increase of pacing threshold caused by fibrosis around the electrode tip, and intravascular migration of severed electrodes. ${ }^{1}$ Major venous thrombosis resulting from endocardial pacing electrodes occurs rarely. The two patients reported here developed a chronic superior vena cava syndrome secondary to progressive thrombosis around the broken transvenous pacemaker electrode. Both had two pacing wires within the cardiovascular system: the new wire and the one that had been implanted previously which was broken and remained firmly attached to the right ventricle with the proximal end lying free in the superior vena cava. The patients showed neither resolution of the thrombus nor recanalisation of the involved vessels after anticoagulant and fibrinolytic treatment. Restoration of adequate venous flow occurred spontaneously with the development of collateral venous channels.

\section{Case reports}

CASE 1

A 54-year-old woman was admitted to the hospital in February 1977 with two months' history of swelling of the face, chest, and arms. The patient had a transvenous pacemaker implanted in December 1974 for sick sinus syndrome. Two weeks Received for publication 9 August 1979 later she underwent an operation because of loss of stimulation. At operation the pacing wire (LEM 603) was found to be damaged near the connection to the pulse generator. An attempt was made to remove the wire, but the distal $24 \mathrm{~cm}$ remained in the right ventricle, right atrium, and superior vena cava. A new pacemaker wire was inserted into the right ventricle through the right external jugular vein. The postoperative course was uneventful and the patient was discharged with a normally functioning pacemaker.

When the patient was readmitted she was complaining of headache and dyspnoea on effort. There was oedema and cyanosis of the face, neck, right arm, and upper part of the chest. The external jugular veins were distended, and venous engorgement was present in the upper arm and the pectoral region. The blood pressure was $140 /$ $80 \mathrm{mmHg}$, and the pulse rate $72 / \mathrm{min}$ and regular. The chest $x$-ray film confirmed the presence of the pacing wire and the broken wire. Blood chemistry was normal except for a prolonged euglobulin lysis time to $320 \mathrm{~min}$ (normal $231 \pm 66 \mathrm{~min}$ ). A clinical diagnosis of occlusion of the superior vena cava was made and heparin (5000 units) was administered intravenously every four hours. There was no improvement within one week of anticoagulation. Venography showed thrombosis in the right and proximal portion of the left innominate vein and the superior portion of the superior vena cava, and there was evidence of collateral circulation through the superficial and deep veins of the neck to the 
azygos vein (Fig. 1a). The patient was placed on a regimen of streptokinase-a starting dose of 250000 units intravenously followed by a maintenance dose of 100000 units/hour over the next 92 hours in a continuous infusion. This produced a satisfactory fibrinolytic state: lysis time was shortened to 10 to 20 minutes. Long-term anticoagulant treatment with heparin was started simultaneously. The drug was subsequently changed to dicoumarol and the treatment was continued. Within 48 hours after streptokinase had been started oedema of the face and neck diminished moderately, but swelling of the right arm remained. Venography was not performed.

In May 1977 she underwent a successful replacement of the pacemaker generator. In December 1977 she was admitted to the hospital for reevaluation. Physical examination showed only slight swelling of the face and arms. Venography showed progressive thrombosis in the superior vena cava extending into both innominate and subclavian veins and the left axillary vein, as compared with the former examination (Fig. 1b). The development of collateral venous channels was also seen. The patient was treated with urokinase, an intravenous loading dose of 7200 CTA units/kg body weight over 10 minutes, followed by a maintenance dose of 3600 CTA units/kg per hour over six hours in a continuous infusion. The treatment was then continued with streptokinase 100000 units/hour over 72 hours, followed by heparin and long-term anticoagulation with dicoumarol and dipyridamole. Recanalisation of major veins was not achieved. The patient has shown mild symptoms for two years of follow-up and has required diuretics twice a week.

\section{CASE 2}

This 72-year-old man was admitted to hospital in November 1978 because of swelling of the face and arms for 10 days. He had a transvenous pacemaker implanted three years before admission for complete heart block. Eleven months before admission there was loss of stimulation because of an increase in the pacing threshold. An attempt was made to remove the electrode which resulted in breakage of the wire. The broken wire remained attached to the right ventricle with the proximal end lying free in the superior vena cava. A new electrode was placed into the right ventricular apex through the right jugular vein. The patient was discharged with a normally functioning pacemaker. On the present admission he complained of headache and dyspnoea on exertion. His face was oedematous and cyanotic, the external jugular veins were distended, and the arms were swollen. Blood pressure was 130/70

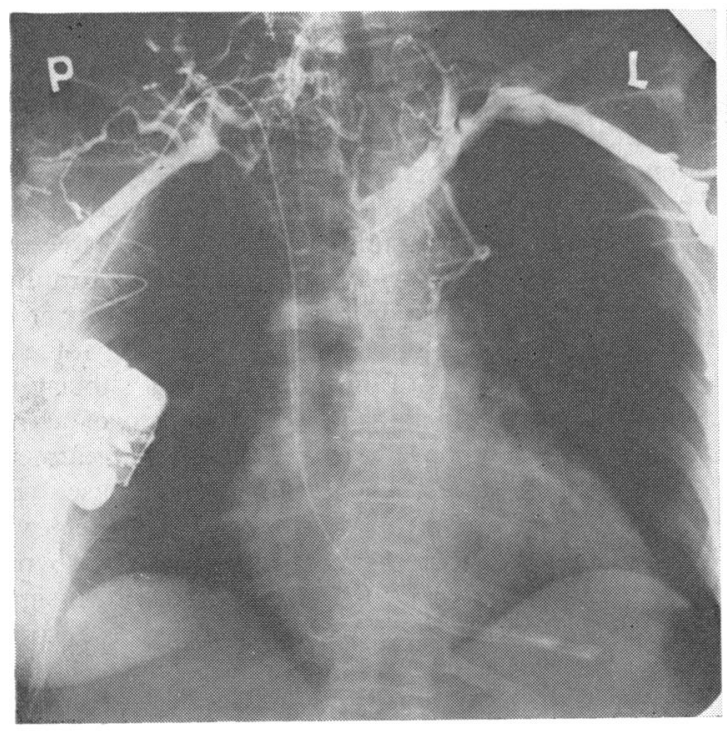

a

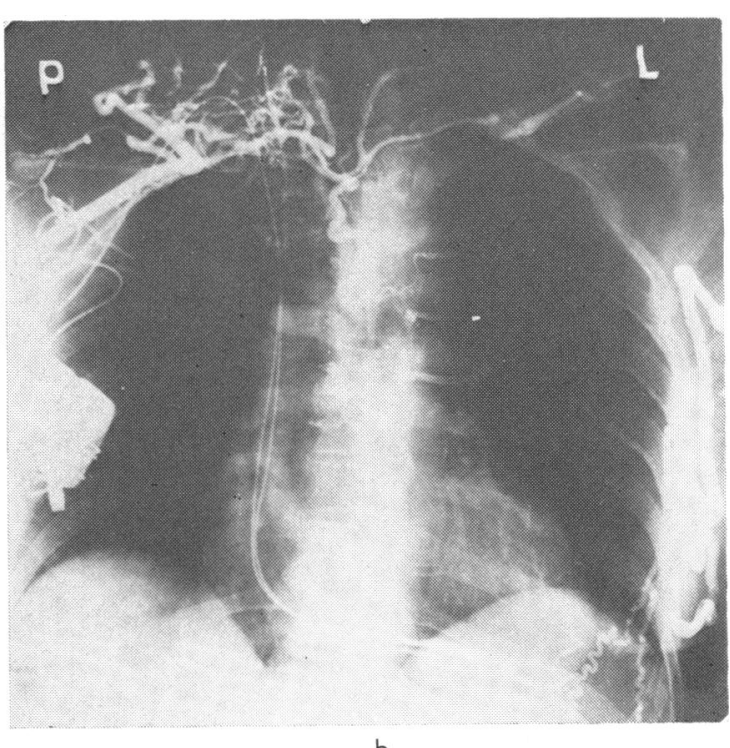

b

Fig. 1 Case 1. (a) Superior venacavogram taken before treatment showing complete occlusion of the superior vena $c$ zva up to the junction of the azygos vein as well as of the right innominate, subclavian, and proximal portion of the left innominate vein. Collateral circulation from the neck veins to the azygos system. (b) Ten months later, occlusion extended into the innominate system, both the subclavians, and the left axillary vein. Venous collateral circulation increased. 
$\mathrm{mmHg}$, and the pulse rate $72 / \mathrm{min}$ and regular. Electrocardiogram showed a normally functioning pacemaker. Chest $x$-ray film showed slight cardiac enlargement and the presence of the working electrode as well as the broken wire. Venography showed complete occlusion of the superior vena cava, innominate system, and proximal portion of the subclavian veins, with multiple collateral veins in the axilla, chest wall, and neck (Fig. 2). The patient was treated with heparin for two days followed by streptokinase in an initial dose of 250000 units and a maintenance dose of 100000 units/hour over 96 hours in a continuous intravenous infusion. During this time oedema of the face and arms decreased. Venography performed three weeks after fibrinolytic treatment showed no recanalisation of the vessels. Collateral circulation was well developed. Long-term anticoagulant treatment has been continued, and the patient has shown mild symptoms for two months of follow-up and has required diuretics once a week.

\section{Discussion}

Thromboembolism is a rare but potentially lifethreatening complication of transvenous cardiac pacemakers. So far only a few cases have been reported, with thrombosis of the axillary, subclavian, and innominate veins or of the superior vena cava, ${ }^{2-4}$ and fatal pulmonary embolism resulting from thrombosis in the right heart and

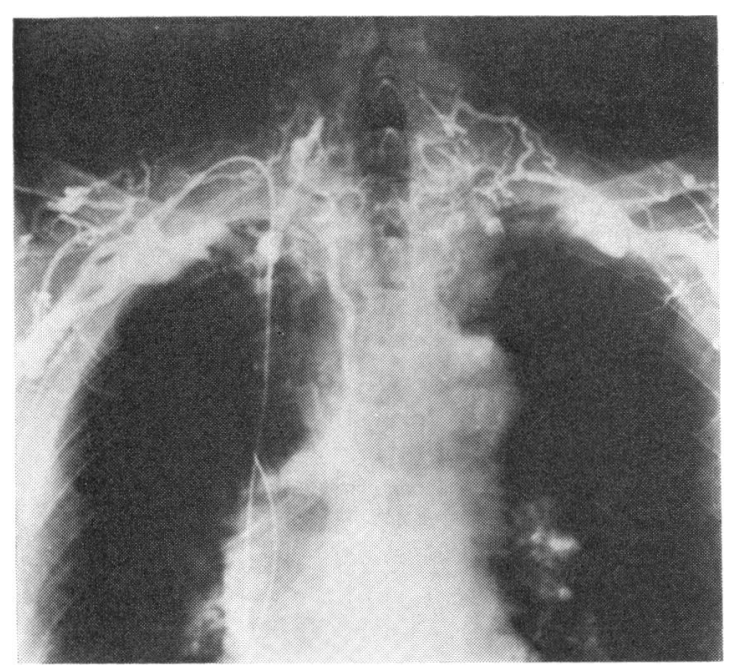

Fig. 2 Case 2. Total occlusion of the superior vena cava, innominate system, and the proximal portion of subclavian veins, with collateral circulation through the neck and chest wall veins. Note the presence of the broken pacemaker wire in the superior vena cava. major veins. ${ }^{5-7}$ Crook et al. ${ }^{8}$ reviewed a series of 125 patients on permanent transvenous pacing and found occlusion of the subclavian vein in only three of these patients. Subclinical thrombosis, however, may occur frequently as a result of pacemaker insertion. A venographic study by Balau et $a l .{ }^{9}$ in 49 patients, after pacemaker insertion through the cephalic vein, showed radiological evidence of thrombi in 14 , but only three of them developed oedema of the arm. A recently reported venography study ${ }^{10}$ in 34 patients showed partial or total obstruction of the innominate or subclavian veins in 22. Collateral circulation was well developed in most of them. The superior vena cava syndrome secondary to thrombosis along the course of the pacing wire may be encountered immediately or as late as 15 months after pacemaker insertion. Most of these patients respond well to heparin or to streptokinase treatment, particularly when recognised early. ${ }^{3} 411$

Our two patients developed symptomatic major venous thrombosis around the broken electrode wire as late as 11 months and three years after pacemaker insertion, respectively. In both instances the remnant of the electrode which we failed to remove served as a continuous trigger for thrombus formation. Damage to the endothelium of the superior vena cava caused by persistent movement of the broken wire firmly attached to the beating heart could have been the major predisposing factor.

Initially, case 1 had thrombotic occlusion of the superior vena cava as well as the right and a short proximal portion of the left innominate vein. Clinical onset, however, was silent and the superior vena cava syndrome developed gradually over two months. Streptokinase treatment instituted at the time of advanced organisation failed to resolve the thrombi. Despite long-term anticoagulant prophylaxis thrombosis was progressive. Venography at 10 months of follow-up revealed extension of the thrombosis into both innominate and subclavian veins and the left axillary vein. Because of active thrombosis another course of fibrinolytic treatment with urokinase followed by streptokinase was administered but recanalisation of the vessels was not achieved. Fibrinolytic treatment was also not effective in the second patient who had shown signs and symptoms of the superior vena cava syndrome for two weeks before streptokinase was started. Obstruction to venous flow, however, was relieved in great part in both patients by the development of collateral circulation.

As experience with fibrinolytic treatment in venous thrombosis has been accumulated it has become clear that this treatment effectively lyses clots of recent origin. Occasionally even clots of 
more than 10 days' existence may be lysed, but the success of treatment is, in general, inversely proportional to the age of the clot. ${ }^{12}$ Successful streptokinase treatment, however, was reported in chronic arterial occlusive disease, provided organisation of thrombi is greatly delayed..$^{13}$ Though treatment with streptokinase carries a risk of excessive bleeding and is generally unsuccessful in long-lasting thrombosis, we applied this form of treatment because of clinical suspicion that the thrombosis was still active, and in the hope of reducing the extension of venous occlusion.

Review of our two cases clearly indicates that there is a definite risk of major venous thrombosis after pacemaker insertion if a broken pacing wire is retained in the cardiovascular system. We believe that all patients with a broken wire, which cannot easily be removed, should be on long-term anticoagulant therapy.

\section{References}

1 Chardack WM. Cardiac pacemakers and heart block. In: Gibbon JH Jr, Sabiston DC Jr, Spencer F, eds. Surgery of the chest. Philadelphia: WB Saunders, 1969: 824.

2 Griepp RB, Daily PO, Shumway NE. Subclavianaxillary vein thrombosis following inplantation of a pacemaker catheter in the internal jugular vein. f Thorac Cardiovasc Surg 1970; 60: 889-92.

3 Friedman SA, Berger N, Cerutti MM, Kosmoski J. Venous thrombosis and permanent cardiac pacing. Am Heart $\mathcal{f}$ 1973; 85: 531-3.

4 Williams DR, Demos NJ. Thrombosis of superior vena cava caused by pacemaker wire and managed with stroptokinase. I Thorac Cardiovasc Surg 1974; 68: 134-7.

5 Prozan GB, Shipley RE, Madding GF, Kennedy PA. Pulmonary embolism in the presence of an endocardiac pacing catheter. $\mathcal{F} A M A$ 1968; 206: 1564-5.

6 Reynolds J, Auslinger D, Yore R, Paine R. Transvenous cardiac pacemeker, mural thrombosis and pulmonary embolism. Am Heart $\mathcal{F}$ 1969; 78: 688-91.

7 Kaulbach MG, Krukonis EE. Pacemaker electrodeinduced thrombosis in the superior vena cava with pulmonary embolization. A complication of pervenous pacing. Am f Cardiol 1970; 26: 205-7.

8 Crook BRM, Gishen P, Robinson CG, Oram S. Occlusion of the subclavian vein associated with cephalic vein pacemaker electrodes. Br F Surg 1977; 64: 329-31.

9 Balau J, Buysch KH, Marx E, Seling A, Knierem HJ. Thrombose der Vena subclavia nach transvenoser Schrittmacherimplantation. Radiologie 1971; 11: 50-3.

10 Horwitz N. Venous obstruction seen to follow pacing. Med Tribune 1976; 32: 17-21.

11 Sethi GK, Bhayana JN, Scott SM. Innominate venous thrombosis: a rare complication of transvenous pacemaker electrodes. Am Heart $\mathcal{f}$ 1974; 87: 770-2.

12 Ockene IS, Dalen JE. The role of fibrinolytic agents in the treatment of thromboembolism. In:Donoso E, Haft J, eds. Current cardiovascular topics. Thrombosis, platelets, anticoagulation and acetylsalicic acid. Vol 2. Stuttgart: Georg Thieme, 1976: 44-59.

13 Martin M, Schoop W, Zietler E. Streptokinase in chronic arterial occlusive disease. $\mathcal{F} A M A$ 1970; 211 : 1169-73.

Requests for reprints to Dr Henryk Krug, Department of Cardiology, Medical Academy, 1-2 Dluga Street, 61-848 Poznan, Poland. 